\title{
EVOLVING SELF-ORGANIZING ACTIVITIES
}

\author{
Addressing Innovation and Unpredictable Environments
}

\author{
Ghada Alaa and Guy Fitzgerald \\ Department of Information Systems and Computing, Brunel University, Uxbridge, Middlesex, \\ $U K$
}

Abstract: Traditional development methods are systematic, prescriptive and plan-driven, and can reduce creativity and the ability to respond in situations of rapid change. In the ere of the Internet and increased uncertainty it is argued that development teams need to be self-organizing, i.e. able to reflect and adapt freely to specific problem situation instead of following rigid methods. A way of operationalizing these concepts is presented and this paper elaborates a case study experience, a business-business (B-B) e-marketplace relating to the pharmaceutical sector, where self-organizing activities have been evolved in modeling upfront requirements for the portal. It is based on brainstorming sessions and the use of a modeling tool, the 'e-Business Issues Roadmap' that categorizes possible issues that such an e-business project might encounter and hence provides a representation or a global 'picture' of the problem situation. The outcome is the trigger of business innovative ideas that are unlikely to have been generated by traditional modeling techniques. By re-visiting this exercise the 'picture' becomes up-to-date and accordingly stakeholders will self-organize to address the necessities of the specific situation. The paper concludes with the introduction of the concept of the 'Practice' that is defined as 'a discourse during which requirements for software development activities and responses evolve'. It helps with the habits and dynamics required to instill self-organization within development teams rather than leaving it to evolve by chance.

Key words: innovation, emergence, complex adaptive systems, self-organization, practice

\section{INTRODUCTION}

The turbulent nature of the business environment (Turban et al., 2000) requires organizations to react quickly and creatively to make the most of 
new opportunities and business models. The globalization effect of the Internet creates pressure on organizations to raise the quality of their products and services as well as react more creatively and quickly to be able to compete internationally. The new development environment is thus characterized by high competitive pressures, large amounts of change, uncertainty and increased time pressures.

Avison and Fitzgerald (2003) point to the current debate and questioning of formal methodologies and characterize this as a 'backlash against methodology'. One element of this backlash is that some developers are rejecting the use of methodologies and turning to less formal more 'ad hoc' development. Highsmith (1998) suggests that the discipline of software engineering is based on rigorous concepts like predictable events, deterministic patterns and linear construction of objects, and that this is insufficient to cope with the high frequency of change and the speed of the market. The limitations of traditional development methods for software innovation and adaptability are discussed in section 2 , and the requirements of innovative and adaptive development processes are addressed in section 3.

Alaa and Fitzgerald (2004) identify the importance of fostering innovative development environments and raising the ability of developers and stakeholders to react and adapt freely to specific problem situations. It is argued that innovation and adaptability can be achieved through selfgovernance and self-organization of their activities. These arguments are based on the theory of complex adaptive systems and the phenomenon of organization emergence that sees the world as a living system, and will be discussed further in section 4 and 5 .

Experience with a B-B e-marketplace case directed to the pharmaceutical industry in Egypt will be elaborated where self-organizing activities in modeling upfront requirements for the portal have been evolved (see section 6). The outcome is the trigger of business-value ideas as a response to specific problem situations. Hence self-organization builds in the ability to respond and adapt freely according to the requirements of the specific situation. The paper concludes with the necessity to instil self-organization within development teams instead of leaving it to evolve by chance, as suggested through the introduction of the concept of the 'Practice'. This is defined as "a discourse during which software development activities and responses evolve" (see section 7). 


\section{LIMITATIONS OF TRADITIONAL METHODOLOGIES FOR INNOVATION AND ADAPTABILITY}

According to Baskerville et al. (1992) the traditional thinking of methodical information systems development is unsuitable for the new software development environment, they argue that new businesses are unpredictable and non-linear whereas traditional development methods are systematic, ordered, regular and regimented and therefore are unable to respond to frequently changing and shifting environments.

De Macro and Lister (1987) state that as traditional methods are rigorous and lessen the use of imagination and creativity. They argue that strict use of methods lead people to concentrate on procedures and documentation rather than the actual product or service. Avison and Fitzgerald (2003) refer to this as 'strict adherence' to the methodology rules that they describe as slavish and inhibiting of creative thinking together with an inability to respond to change.

Traditional methodical thinking is typically oriented towards large-scale, long-term, software projects that have long-term objectives (Baskerville et al., 1992) but in a world involving frequent, radical changes, having processes as repeatable and optimized is like a 'Dinosaur' (Highsmith, 2000) and will lead to software failure. Therefore Highsmith (2000) suggests in order to succeed in the post-modern globalization of commerce and industry rapid change-tolerant development processes aiming at short-term needs rather than long-term investment for the future is critical to enable organizations to adapt quickly to their environments.

Development methodologies according to Avison and Fitzgerald (2003) can also be inflexible, not allowing changes to requirements during development, as well as 'one-dimensional'. Further they argue that a universal development approach may not address all particular organization situations. Recently new technologies (end user computing, fourth generation languages and local area networks) have also brought incremental, fragmented, non-linear development (Baskerville et al., 1992). These new technical innovations will lead to the development of fragmented systems elements in unique ways that contradict the assumptions of traditional methodical thinking.

Therefore new practices and processes for information systems development are required to produce fast changing innovative information systems able to adapt to an ever-changing environment. 


\section{REQUIREMENTS OF INNOVATIVE AND ADAPTIVE DEVELOPMENT PROCESSES}

According to Riehle (2000) the traditional practice of software development regards the development process as something that has to be planned and controlled in order to reliably achieve the desired result. However, Highsmith (1997) argues that as industries move from predictable, stable environments to unpredictable, nonlinear and fast-changing environments, traditional information systems development practices based on determinism, predictability and stability will be inadequate to foster innovative development. Therefore according to Highsmith (1998) the basic tenants of process improvement, software engineering, and command and control management, are unsuitable.

Traditional rigorous methods according to Baskerville et al. (1992) are based on rational scientific reasoning that assume empirically testable propositions that in turn give a 'truth value'. Highsmith and Cockburn (2002) indicate that traditional methodologies provide what they call 'allinclusive rules', i.e. strict steps or procedures to be applied under all situations, depending on voluminous written rules that will apply for every situation. Instead software developers need to depend on their creativity to respond to diverse circumstances.

Highsmith (1997) believes that RAD practices, that involve evolutionary development, customer focus groups, JAD sessions, technical reviews, timeboxed project management and continuous software development, are a step in the right direction to achieve innovation and adaptability. This is because they establish innovative collaborative environments and ensure the fast movement and feedback necessary to adapt to change. Riehle (2000) explains that evolutionary prototyping views software development as a shared learning experience based on requirements negotiation and timely feedback from customers, which foster creativity and innovation, as well as accommodating changes in markets.

But according to Truex et al. (1999) development frameworks aiming at rapid and flexible development such as prototyping, end-user development, open systems connectivity and contingent approaches are still insufficient for software emergence, as they still aim to achieve a stable product. They explain that a low-maintenance, stable IT system will battle against the everchanging environment instead of adapting to it, and that this will inhibit rather than facilitate organizational emergence. Assuming stability will freeze the organizational change instead of achieving continuous change and fluidity (Truex et al, 1999).

Truex et al. (2000) argue that although different development methods apply different techniques, sequences, steps and activities, but despite this 
seeming diversity they have common assumptions and idealized characteristics. The definition they use for 'method' is the one given by Oxford and Webster's dictionaries; the primary definition given is 'a method is the procedure of obtaining an object', and the secondary definitions describe the method as 'orderly', 'systematic', 'regularity' and 'regimen'.

They suggest instead a shift in the development mode from structured, orderly and methodical to unstructured, emergent and amethodical to fulfill the requirements set by the new development environments. By amethodical they don't mean a descent into chaos and anarchy, but emancipation from structure and regularity. Amethodical systems thinking implies orchestration of the development activities but not a predefined sequence, rationality or universality. Thus, amethodical views of information systems development supports conflict over consensus and forces the developers to attend to the different voices and interests and not seek simply a compromise solution.

The amethodical view also appreciates innovation and 'organizational shake-ups' that lead to adaptation, experimentation and in turn to accidents and opportunism. Truex et al. (2000) outline the differences between methodical and amethodical information systems development, by characterizing the methodical view as controlled, linear, universal and rational, and the amethodical view as random, non-linear, unique (occurs in completely unique and idiographic forms) and capricious.

This paper agrees with these arguments and adopts new assumptions based on social construction and autopoiesis. In the following section we examine the phenomenon of organization emergence which provides the theoretical underpinning of how development processes emerge as response to specific problem situations.

\section{ORGANIZATION EMERGENCE}

Organization emergence according to Baskerville et al. (1992) is characterised as the organization being in continual change, following no predefined pattern and never reaching a steady state. The organization may in fact exhibit temporal regularities, but it remains constantly in transition without ever becoming fully formed. Hence the theory of emergence rejects the notion of structures, regularities and stability. In the following we examine the phenomenon of emergence and its properties.

\subsection{The Theory Behind Emergence}

According to Riehle (2000) high speed and high change of the market make businesses unpredictable and hence unplannable in the traditional 
sense of control and optimization. Highsmith (1997) argues that high speed and frequent change induces complexity that has more to do with the number of interacting agents, and the speed with which those agents interact, rather than with size and technological complexity. Today's organizations involve many independent agents; customers, vendors, competitors, stockholders interacting with each other, at a speed that implies that linear cause-andeffect rules are no longer sufficient for success.

Highsmith (1998) stresses the need for a new way of thinking about how complex software products come into being. He believes that by studying life itself we can adopt mechanisms for self-production and evolution. He compares information systems to a self-contained, enclosed, living ecosystem. Kelly (1994) explains how in the dawn of the new era systems become so complex and autonomous as to be indistinguishable from living organisms. He provides a reasoning of evolving complex systems instead of engineering them by looking to them as self-sustaining living systems.

Complex Adaptive Systems (CAS) theory (Highsmith, 1997) provides three main concepts to explain the world: agents, environments, and emergent outcomes. Agents compete and cooperate to get work done, but the final result is not the outcome of the work of any particular agent or process. Effectively, the result emerges from the overall competition and cooperation of the agents, therefore system behavior cannot be predicted because simple cause-and-effect reasoning has broken down. Therefore Highsmith (1997) argues that the absence of group behavior rules within organizations is a good phenomenon as there are no more algorithms or procedures that define the results expected from the group, hence the group behavior emerges, rather than being predefined.

\subsection{Emergence is not just a notion of Change}

Change is a transition over time from one state to another, but emergence is about evolution, which means emergent organizations are always in the process of "remaking" themselves, never reaching a steady state. Therefore adaptation in the 'Internet-time' environment is not just another word for change management or following a plan (Highsmith, 1998).

\subsection{Emergence is not just Contingency}

Contingency approaches to information systems development are an attempt to introduce flexibility into the systems development process. However, the contingency approach still provides a structure or a framework that guides the developers in choosing the appropriate techniques and tools according to the specific situation. In emergent environments selecting a 
path from an almost unlimited number of alternate paths will freeze the organization's assumptions about itself and its environment, which is argued to contradict and inhibit emergent behavior. The produced plan will become out of date very quickly and, rather than focusing on the outdated plan, it is important to deal with the changing realities directly (Highsmith and Cockburn, 2002).

\subsection{Adaptability through Spontaneous Order not Predictability}

Predictability and plan-driven strategies result in rigidity and are thus less able to respond to change, and according to Highsmith (1998) process improvement or change control management activities, based on predicted outcomes, negating our product development efforts. Schwaber (2002) makes clear that team structures and requirements emerge during the course of the project rather than being determined at its outset, therefore it is critical for organizations to adapt quickly to their environments rather than tending to create a fit between the information system and the existing structure. Baskerville et al. (1992) talk about 'seizing the opportunity' to develop the necessary organizational flexibility to cope with the situation and its possible opportunities based on small-scale and short-term planning. But of course the team is guided by preliminary and sketchy visions of requirements and architecture, not planning for long-term outcomes.

Having examined the notion of organization emergence, or as Highsmith (1998) terms it, the 'spontaneous order', we now look at how this might be achieved. In the following section we explore ways to operationalize these concepts to become more creative and adaptive to change.

\section{SELF-ORGANIZATION FOR INNOVATIVE AND UNPREDICTABLE ENVIRONMENTS}

As mentioned above Highsmith (1997) finds that a growing number of software projects are so complex that the development processes are inherently unplannable, yet successful products can emerge from such environments. He argues that creative group behavior emerges unpredictably from spontaneous self-organization. Vidgen et al. (2002) also note that the success of OSS (Open Source Software) is superficially surprising, in fact they suggest that OSS is a triumph of self-organization, rather than hierarchical regulation. Kelly (1994) also points to self-organization as the essence of innovation and the way to cope with business turbulence. Riehle 
(2000) concurs and argues that the underlying assumption of equating developers performing the development process to computers executing software is not appropriate, instead it is suggested that self-organizing teams lead to an environment in which innovation and adaptation thrive so that 'local order' can emerge.

Highsmith (1998) defines self-organization as a property similar to a collective "aha," that moment of creative energy when the solution to some nagging problem emerges. Such self-organization arises when independent individuals cooperate to respond creatively to, and reflect on, a specific problem situation. Truex et al. (1999) argue that self-organization is not deterministic, rather a product of a constant social negotiation, continual change of work culture and decision processes where outcome stages arise from previous history and context. This they refer to as the 'dialectics of organizational autopoiesis'. Hence self-organization refers to a theory of social underpinning derived from the unstable environment in which the information system will be developed. The reasoning of autopoietic, or selfreferential social systems, lies in social organizations that are continuously self-making via discourse, that will never reach a steady state (Baskerville et al., 1992).

Riehle (2000) indicates that this distinction implies that different development processes are required. The differences focus upon; how a development methodology views customers, how it fosters creativity and innovation, how it tolerates changes in the market and in requirements. For example RAD practices are characterized, according to Fitzgerald (1998), by active user involvement and small-empowered teams, which in turn supports the reasoning of self-organization. In fact the focus on JAD sessions, incremental prototyping and focus groups, engage the development team members and the stakeholders to collaborate and jointly innovate the product solution. But still RAD follows a rigid agenda and uses a traditional topdown approach, often supported by an integrated CASE tool, which reduces creativity and the ability to respond freely to change. On the other hand OSS (Open Source Software) developers have been identified as self-selected, highly motivated and self-deprecating, which has important implications for stimulating co-operative development in order to realize complex and mission-critical software such as operating systems, email servers etc. with less people, within time, within budget and with high quality (Feller and Fitzgerald, 2000) (Vidgen et al., 2002).

Riehle (2000) stresses that self-organization cannot be commanded but must be nurtured. Kelly (1994) points to the need to instill in organizations guidelines and self-governance and relinquish what he calls 'our total control'. In the following a case study experience is explored, a businessbusiness (B-B) e-marketplace, where self-organizing activities in modeling 
upfront requirements for the portal have been evolved as a way to respond to the turbulent and unpredictable settings that required high degree of innovation and responsiveness. The findings of the case study pose the question of how to provide mechanisms and dynamics to instil selforganization instead of leaving it to evolve by chance. This research is still ongoing and those questions need to be addressed in our further studies.

\section{EVOLVING SELF-ORGANIZING ACTIVITIES IN REQUIREMENTS MODELING: A CASE STUDY EXPERIENCE}

\subsection{The Case Situation and Research Methodology}

A study of a B-B (business to business) e-marketplace application directed to the pharmaceutical industry in Egypt has been carried out. The primary objectives were to explore practical issues in adopting development methodologies within real-case complex and turbulent settings, identify problems and if required suggest new approaches. A portal application had already been developed before the fieldwork was carried out. The fieldwork of this study was initially part of an evaluation of the project, as it was decided that the application needed to be changed/adjusted after its launch to improve its competitive value. The original analysis and design of the emarketplace identified the functional building blocks of the pharmaceutical trading supply chain.

According to Baskerville and Wood-Harper (1996) the purpose of 'action research' is to observe and create effective organizational change and they consider it ideal for studying new or changed systems development methodologies. The complex, multivariate settings of the e-marketplace made problem diagnosis efforts as well as intervention difficult and not straightforward. Susman and Evered (1978) state that in terms of generating action and knowledge, action research employs a 'process view' of research and they provide a model of the process as a five phase cyclical process; comprising diagnosing, action planning, action taking, evaluating and specifying learning. These processes were adopted and the phases indicated below. Firstly, the research method elements of the case are highlighted and this is followed by the content and discussion elements.

During 4 months of case study work the researcher identified problems resulting from the developers having become overwhelmed with too much detail during the comprehensive traditional analysis of the e-business case. For example, the order lifecycle was decomposed into order preparation, 
order placement and order fulfillment, each in turn being extensively analyzed. As a response the researcher produced an overview of the major functional elements of the trading process to replace the detailed flowchart decompositions. For example, the following major functional elements were outlined; fixed price purchasing, special offers, return of expired products, billing and invoicing, online payment and delivery tracking. (Diagnosing Phase)

As a way to evaluate and re-define requirements for the portal we identified the importance of what we termed 'random upfront' requirements modeling and design where global strategic requirements and design features can be developed spontaneously. For us the term 'upfront' does not mean 'initial' as the application will undergo continuous, constant development as indicated by Truex et al. (1999) and therefore there is a need to go back again and again to review the global requirements. Of course there will be a need to carry out a comprehensive analysis and to get to the level of detail needed to build the application, but this comes after the proposed 'spontaneous upfront' analysis exercise. (Action Planning Phase)

As an aid to analysis a modelling tool designed for e-business projects, the 'e-Business Issues Roadmap' (Figure 1) was introduced. This provides a model of the problem situation and by re-visiting it stakeholders identified problems and issues that emerged over time and accordingly self-organized to the needs of the problem situation. Three user-based analysis sessions were undertaken to try out the modelling tool based on brainstorming sessions and as response to that design ideas and/or appropriate actions were triggered to address the specific problems faced. As the objectives were to model upfront strategic issues only a few such sessions were needed. (Action Taking Phase)

The source of data collected for the research was primarily interviews (unstructured at the beginning then semi-structured) conducted mainly with the business consultant and the IT manager, but also with the general manager, the business analyst, members of the IT and technical staff, the legal representative and the users (pharmacists, pharmaceutical companies and distributors). A total of fifteen interviews were conducted. The other sources of data included meetings (a total of seven), observational notes, documents and protocols of the application.

Afterwards two additional meetings were held with the general manager and the IT manager to discuss ways to operationalize some of the design ideas and actions produced from the proposed upfront exercise. Their response was very positive about the business-value of the ideas triggered. (Evaluation Phase) This research work is ongoing and further work is required to improve the reasoning of self-organization within development 
processes and analysis of benefits, insights and drawbacks associated with its use. (Specifying Learning Phase)

\subsection{The Process Characteristics and Outcomes}

To identify and analyze innovative requirements a heuristic approach is used as it is more appropriate than a rigorous approach, we initially thought brainstorming sessions would be appropriate for this purpose. Joint Requirements Planning (JRP) as well as Joint Application Development (JAD) sessions (Martin, 1991) have been suggested as a means to set up the initial requirements for a project through brainstorming activities, but we feel they are too narrowly focused. They concentrate on producing detailed requirements and artifacts such as functional decomposition diagrams, dependency or data flow diagrams, etc. They are also highly structured and involve formal meetings that have defined rules of behavior e.g. the use of well-defined agendas, keeping official meeting minutes, the involvement of a qualified facilitator, etc. These we felt unlikely to engender the necessary creativity and found agile modeling sessions to be more appropriate.

Ambler (2001b) suggests that agile modeling sessions be highly iterative, with the agile modeler iterating back and forth; as it is more common to identify a requirement, analyze it, and propose a potential design strategy within minutes, hence the need to iterate quickly between phases. Also the formulation of requirements is likely to happen by asking questions, therefore Ambler (2001a) suggests the use of user stories (Beck, 2000) that are essentially very high-level wish list of stakeholders. Ambler (2001a) characterizes user stories as a reminder to conduct a conversation with project stakeholders and capture high-level requirements, including business rules, constraints, and technical requirements.

However, for internet-based applications we believe that the complexity of such applications, together with the variety of multi-disciplinary issues that interplay, make the use of user stories too vague and loose. Therefore the 'e-Business Issues Roadmap' (Figure 1), mentioned above, was derived (Alaa and Stockman, 2001). This provides a template that categorizes the different underlying issues and concerns that typical internet-based projects face. The classification proposes issues by sector, e.g. business, technological, legal, political, economic and social, and the level they work at, for example, intra-organizational, customers and partners and environmental. Due to the complexity and the large variety of potential issues, the proposed roadmap acts as a 'balance' between structure and freedom by providing an initial set of topics/issues that need to be thought about. It is used to drive and guide the triggering of issues under the various identified categories but still leaves space to the modeler to be creative. The 
Roadmap provides a starting point without which some of the many relevant issues might be missed and too much time wasted but it is not restrictive as other issues are readily triggered by discussion and added.

This resulted in the running of the three modeling sessions. Only three were undertaken because a comprehensive analysis was not required, just an up-front set was sought. This also helped to reduce complexity and stimulate creative thinking. As the underlying issues are multi-disciplinary, the participants of the modeling sessions included representatives of the different kinds of members (stakeholders) involved with operationalizing the e-marketplace portal.

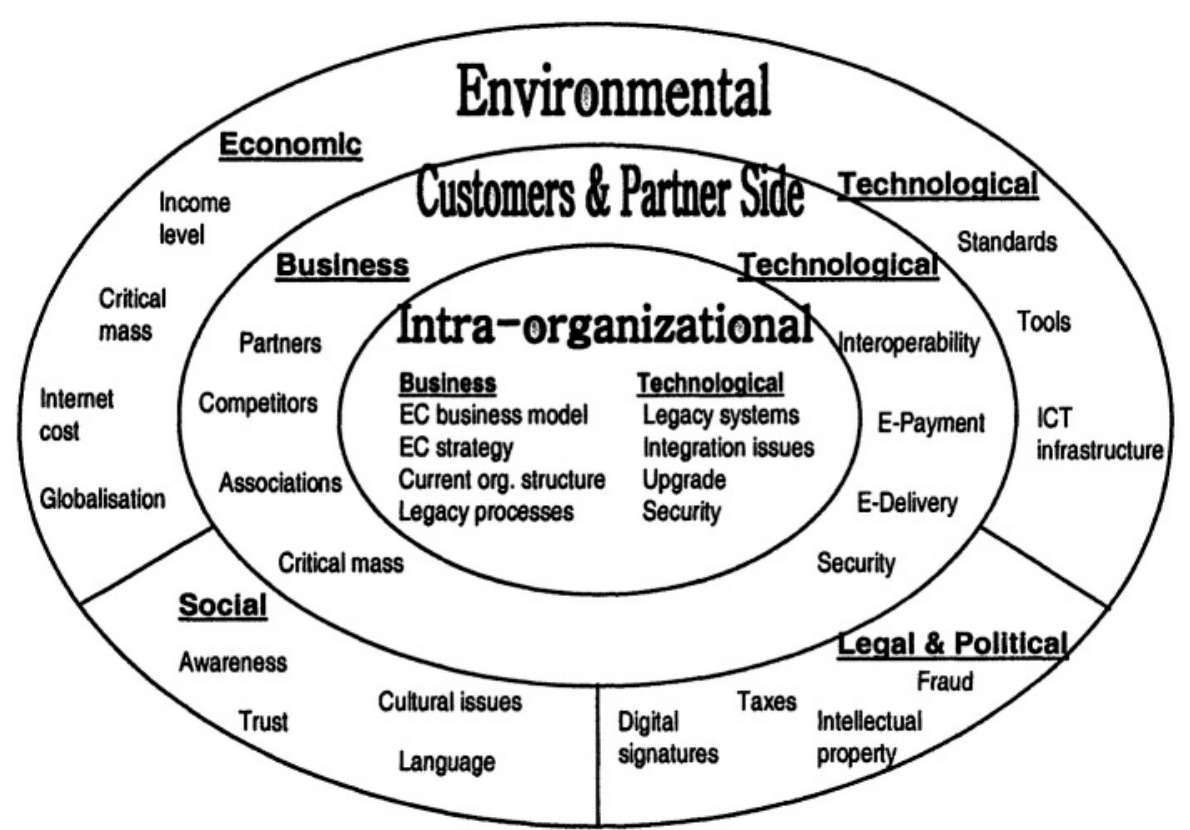

Figure 1. e-Business Issues Roadmap

The first brainstorming session was attended by the business consultant, the business analyst and the researcher where issues were triggered under the different identified categories in the roadmap and consequently the implications of the issue and possible solutions and drawbacks were discussed. One of the issues triggered in this session was that the organizational structure needed to suit the e-marketplace architecture and not vice versa. Another was that a sales pipeline was identified as necessary to fulfill the sales process, as some of the major activities were still carried out offline. Some of the solutions triggered in this session were the 'awareness campaigns', the 'installment program' and the different value-added 
services, such as the customization of product dispatch as part of the order placement cycle (Figure 2).

There was a need to hold other sessions with representatives of the technical staff as well as senior management to take the different issues perspectives into consideration. So another brainstorming session was held and some of the issues that came out in this session were that the e-trading process is invisible and ambiguous over the traditional one, which it was felt might make the buyers reluctant to use the e-marketplace application. This resulted in a focus on the importance of the confirmation of the order before it is placed. Another idea was to provide virus-shield software as a way to further increase customer trust and satisfaction. These and other ideas are shown in Figure 2.

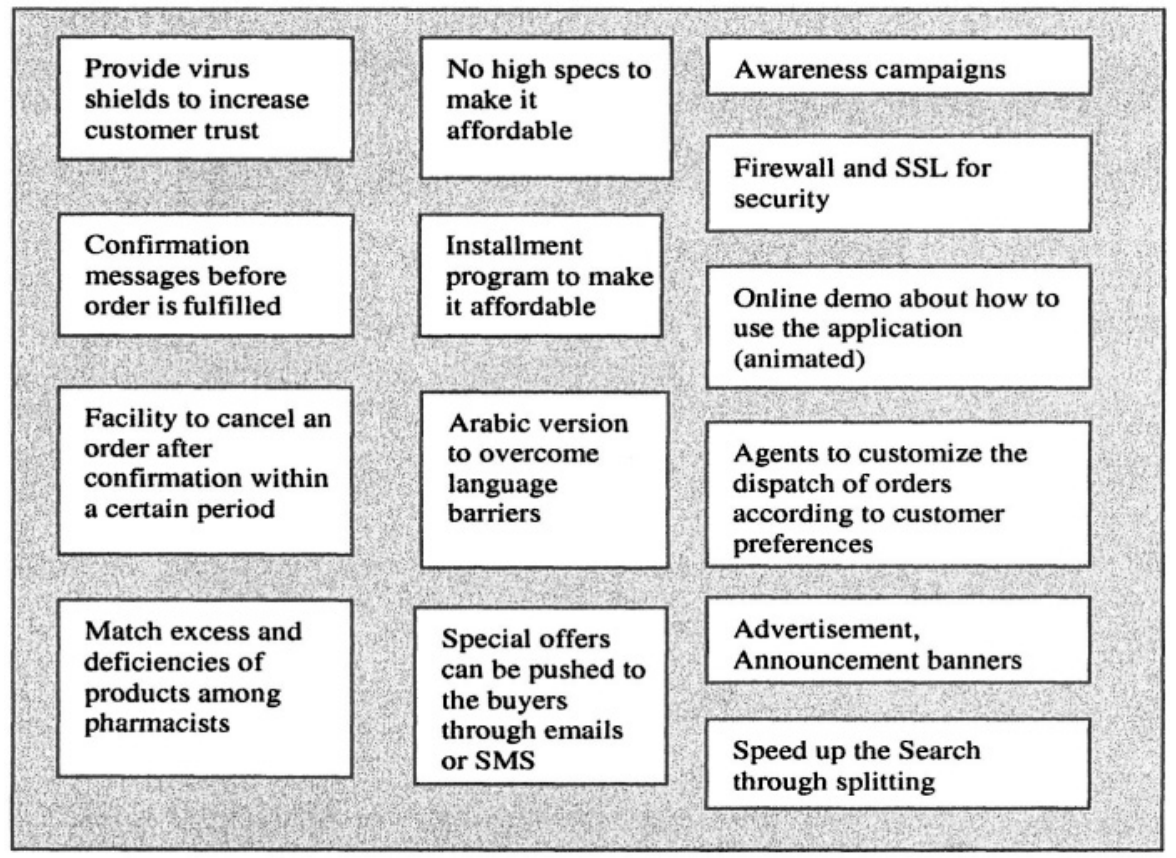

Figure 2. Design Ideas and Actions Triggered

Another session was arranged later with the general manager, the business consultant and the researcher where the attendees started to reflect on and evaluate (in an informal way) these ideas/solutions (Figure 2). The ideas they really liked were the focus on value-added services to further publicize the site and providing virus shield software. Using agent-based software was postponed for the next release, as the company wanted to make some revenue before undertaking further investment. Other ideas were hard to implement, as the current system architecture would not allow this easily, 
such as the customization of the order preparation function, which would necessitate the redesign of the order module.

This is an example of how the proposed 'spontaneous upfront modeling' has been applied. The resulting design ideas and actions (Figure 2) show a number of new and innovative features for the e-business application, e.g. SMS messaging of special offers, the introduction of an installment program, etc. that were not identified before and did not emerge from the traditional functional decompositions and use cases. The 'e-Business Issues Roadmap' provides a sort of a global picture of the whole situation, and by visiting the proposed exercise in a periodical basis will keep the 'picture' updated. This helps to respond to unpredictable problems and issues that emerge over time, in this way project stakeholders self-organize according to the implications of the problem situation. A more detailed description and derivation of the approach are provided in Alaa and Fitzgerald (2004).

\section{TOWARDS 'THE PRACTICE': THE DEVELOPMENT OF SELF-ORGANIZING ACTIVITIES}

The problems encountered in the case by the initial use of rigorous analysis techniques focused attention on the limitations of these traditional techniques. Their systematic, prescriptive and plan-driven nature appeared to reduce creative thinking and the ability to respond to change. The authors proposed approach, which is argued to enhance creativity and spontaneity, is quite different. This leads us not to use the term 'technique' for it, as it relies on people to drive it according to the opportunities and events that arise, rather than following, or being driven by it. We thus call it a 'Practice'. The dictionary definition of the word 'practice' relates to exercise, training, run through, habit or ritual. Applying these meanings to requirements for software development, we identify the proposed 'Practice' as a 'discourse during which software development activities and responses evolve'.

Unlike traditional requirements and software development techniques, the notion of a 'Practice' will support developers in a self-organizing way to create innovative development environments driven by opportunity and events. Therefore the 'Practice' is neither prescriptive, nor anchored in rationality, reductionism, empirical or scientific underpinning. However, some initial rules or guidelines are required to frame the activities, such as the 'e-Business Issues Roadmap' (Figure 1) which sets an initial boundary but leaving space to the development team to react independently to the problem situation. Hence the 'Practice' depends on the individuals and their 
creativity to find ways to solve problems as they arise, rather than providing them with inclusive rules, i.e. all things they could possibly do under all situations.

The 'Practice' can be compared to playing a non-chance 'game' where the general game rules are known, but the game path evolves in use according to the situation and the responses. To win a game you need to understand the game rules and have the appropriate skills, then through 'practicing' the game you will become more skilful and more likely to win. Whereas the 'technique' can be compared to 'cooking instructions or a recipe', that has to be followed strictly in order to get the meal done. Any flaw in the instructions or the ingredients will spoil the meal and its taste.

However, the concept of the 'Practice' is not introduced to replace the 'Technique' nor to find a kind of a compromise, as we believe that they are complementary. The 'Practice' will address habits of the development team and dynamics such as agile modeling sessions and continuously modeling upfront requirements to help stakeholders respond creatively in a rapid, emergent way. Whereas later the 'Techniques' will be needed to carry out a rigorous detailed analysis and design to get to the level of detail needed to build the application.

The concept of the 'Practice' builds also on the requirements of innovative approaches suggested in the literature (section 3). It supports the amethodical view suggested by Truex et al. (2000). This requires development processes to be unstructured, opportunistic and driven by accident. It also builds on the AM (Agile Modeling) methodology suggested by Ambler (2001 a), in fact it fills in some of the gaps of AM that provides only a set of guidelines such as the importance of active stakeholder participation, applying the right artifacts, creating several models in parallel and others (Ambler, 2001 c) without providing a mechanism to operationalize them.

We conclude that the 'Practice' is an exercise carried out by the development team. Unlike the technique, the 'Practice' is a creative and communicative process. It doesn't follow a set of all-inclusive rules; it rather acts as a 'parable' that guides the participants based on a set of concepts, guidelines, action/interaction medium, previous experiences and best practices, and then the 'development game' evolves in use. This research is still ongoing and further studies will address elements and dynamics of the 'Practice' that will further instill and cultivate self-organization within organizations instead of leaving it to evolve by chance. 


\section{CONCLUSIONS}

A highly turbulent business environment and rapid technological developments require a high degree of innovation and responsiveness. The proposed 'spontaneous upfront modeling practice' facilitates business innovation and emergence through informal interaction and global strategic reasoning, about the required solution. It helps by providing the guidance of a non-prescriptive, stimulating requirements modeling tool, the 'e-Business Issues Roadmap', to identify issues, resolve contradictions and overlaps within the e-business project settings and as response to that trigger possible solutions and design ideas according to what the specific situation implies. This is a creative and communicative process that may have to be visited a number of times in order to continuously adapt to ever-changing shifting environments. This is an example of how development teams can selforganize according to specific problem situation instead of following a change plan. Self-organization can be instilled and cultivated within organizations as suggested through 'practicing' or something like 'exercising' that will develop/train the skills needed to maneuver, improvise and innovate, instead of leaving it to evolve by chance.

\section{REFERENCES}

Alaa G., Fitzgerald G., 2004, A Proposal of an Upfront Requirements Modeling and Design Practice for e-Commerce Projects, Proceedings of the Thirty-Seventh Annual Hawaii International Conference on Systems Sciences, January 2004

Alaa, G. and T. Stockman, 2001. An Investigation into the Nature of E-commerce, Requirements and Needs in Developing Countries, BITWORLD 2001 Proceedings, Cairo, Egypt.

Ambler, S., 2001, Agile Requirements Modeling, The Official Agile Modeling (AM) Site, Copyright 2001-2002, http://www.agilemodeling.com/essays/agileRequirements.htm

Ambler, S., 2001, Rethinking Modeling Sessions, The Official Agile Modeling (AM) Site, Copyright 2001-2002, http://www.agilemodeling.com/essays/modelingSessions.htm

Ambler, S., 2001, The Practices of Agile Modeling, The Official Agile Modeling (AM) Site, Copyright 2001-2002, http://www.agilemodeling.com/practices.htm

Avison D., Fitzgerald G., 2003, Where Now for Development Methodologies?Communications of the ACM, January 2003, Vol. 46, No.1

Baskerville R., Travis J., Truex D., 1992, Systems Without Method: The Impact of New Technologies on Information Systems Development Projects, IFIP Transactions A-8 North-Holland 1992

Baskerville R., Wood-Harper T., 1996, A critical perspective on action research as a method for information systems research, Journal of Information Technology (1996) 11, 235-246

Beck, K., 2000, Extreme Programming Explained: Embrace Change, Addison Wesley

Feller J., Fitzgerald B., 2000, A Framework Analysis of the Open Source Development Paradigm, in W. Orlikowski, P. Weill, S. Ang and H. Krcmar (eds) Proceedings of 21st 
Annual International Conference on Information Systems, Brisbane, Australia, December 2000

Fitzgerald B., 1998, A Preliminary Investigation of Rapid Application Development in Practice, Proceedings of 6th International Conference on Information Systems Methodologies, editors Wood-Harper AT, Jayarantna N., Wood J R G, pp. 77-87

Highsmith, J., 1997, Messy, Exciting, and Anxiety-Ridden: Adaptive Software Development, Cutter Information Corp., http://www.jimhighsmith.com/articles/messy.htm

Highsmith, J., 1998, Order for Free, Life - the Artificial and the Real, Software Development, http://www.jimhighsmith.com/articles/order.html

Highsmith, J., 2000, Retiring Lifecycle Dinosaurs, Software Testing and Quality Engineering, July/August pp 22-28

Highsmith J. and Cockburn A., 2002, Agile Software Development: The Business of Innovation, IEEE Software, September/October 2002

Kelly K., 1994, Out of Control: The New Biology of Machines, Social Systems and the Economic World, London : Fourth Estate

Martin J., 1991, Rapid Application Development, Macmillan Publishing, New York

Riehle D., 2000, A Comparion of the Value Systems of Adaptive Software Development and Extreme Programming: How Methodologies May Learn from Each Other, Proceedings of the First International Conference on Extreme Programming and Flexible Processes in Software Engineering (XP 2000). Page 35-50.

Schwaber K., 2002, The Impact of Agile Processes on Requirements Engineering, International Workshop on Time-Constrained Requirements Engineering 2002

Susman, G. I. and Evered, R. D. ,1978, An Assessment of the Scientific Merits of Action Research, Administrative Science Quarterly, Vol. 23, pp. 582-603.

Truex D., Baskerville R. and Klein H., 1999, Growing Systems in Emergent Organizations, Communications of the ACM, August 1999, Vol. 42 No.8

Truex D., Baskerville R., Travis J., 2000, Amethodical Systems Development: The Deferred Meaning of Systems Development Methods, Accounting Management and Information Technology 10: 53-79

Turban, E., Lee, J., King, D. and Chung H. 2000. Electronic Commerce, a managerial perspective, New Jersey, Prentice Hall.

Vidgen R., D. Avison, B. Wood, T. Wood Harper, 2002, Developing Web Information Systems, Butterworth-Heinemann Information Systems Series, Elsevier Science 